\title{
COMMENTARY
}

\section{Do recruitment maneuvers simply improve oxygenation?}

\author{
Franco Valenza* \\ See related research by Constantin et al., http://ccforum.com/content/14/2/R76
}

\begin{abstract}
Recruitment maneuvers have been the subject of intense investigation. Their role in the acute care setting is debated given the lack of information on their influence on clinical outcomes. Oxygenation improvement is often a striking effect, together with changes of respiratory mechanics. However, hemodynamic compromise is frequently associated with the maneuver, sometimes even barotrauma. Another possible downside is bacterial translocation secondary to lung overdistention, as suggested by experimental and initial clinical data. When a recruitment maneuver is performed, the pathophysiological consequences of lung recruitment should guide clinicians more than oxygenation improvement alone.
\end{abstract}

The investigation reported by Constantin and colleagues in a recent issue of Critical Care [1] dwells on a philosophy summarized by Lachmann years ago in an aphorism still current among intensive care practitioners: 'Open up the lung and keep it open' [2]. Along the learning curve of the ventilator settings of patients with acute lung injury, the pivotal role of positive endexpiratory pressure (PEEP) soon became clear. It took some time to understand that PEEP maintains the lung open once, and if, previously opened. Since then several investigators have focused on the recruitment maneuver, proposing different strategies with the common rationale of needing to 'open up' the lung. The study by Constantin and colleagues is one such investigation: their randomized controlled trial aimed to evaluate improvement of oxygenation after a recruitment maneuver performed soon after intubation in the ICU. The setting is new and

*Correspondence: franco.valenza@unimi.it

Università degli Studi di Milano, Fondazione IRCCS Policlinico Ca'Granda, Via F. Sforza 35, 20122 Milano, Italy of interest, although the primary end-point (oxygenation improvement) is somewhat expected in the early stages of acute respiratory distress syndrome, when lung atelectasis is likely to occur [3].

The absence of patients with chronic obstructive pulmonary disease or cardiogenic shock, and the exclusion of patients with encephalopathy, coma, acute brain injury, the need for cardiac resuscitation, or recent thoracic surgery do not allow generalization of the results in the ICU setting. Nevertheless, the trial clearly confirms the culprit role of recruitment maneuvers in the acute care settings.

However, the results of the trial and the discussion of the results raise a number of questions, one of which is: should we care about oxygenation during recruitment maneuvers? Yes, of course. In fact, apart from being a great relief to clinicians, oxygenation improvement usually implies resolution of atelectasis, better distribution of ventilation, and delivery of a given tidal volume to a greater lung volume, hence lower lung strain, all factors that may contribute to attenuate ventilator-induced lung injury. However, oxygenation per se is possibly the least relevant variable. Arterial partial pressure of oxygen $\left(\mathrm{PaO}_{2}\right)$ is only one factor (not the most important for quantity) contributing to oxygen delivery. Is a $\mathrm{PaO}_{2}$ of $80 \mathrm{mmHg}$ a real concern in a sedated paralyzed patient, with normal oxygen carrier capability (hemoglobin) and peripheral delivery of oxygen (cardiac output, distribution of flow)? Not from a metabolic point of view. In this light, the increased $\mathrm{CO}_{2}$ wash-out that followed the recruitment maneuver in Constantin and colleagues' investigation looks more interesting. Unless exceedingly low, what matters is not the absolute value of $\mathrm{PaO}_{2}$, but rather the full price to obtain it: low or high inspiratory fraction of oxygen, 5 or $15 \mathrm{cmH}_{2} \mathrm{O}$ of PEEP, supine or prone position? Thus are recruitment maneuvers. They undoubtedly improve oxygenation in many circumstances, but clearly have a price: frequently hemodynamic compromise, although transient and short lasting; sometimes desaturation; sometimes new air leaks [4].

In their investigation, Constantin and colleagues shed some light on an interesting potential downside of 
recruitment maneuvers: microbiological safety. Data show that mechanical ventilation strategies promote dissemination of intratracheally instilled bacteria [5] or increase susceptibility to development of bacteremia [6]. Constantin and colleagues investigated the relationship between tracheal intubation and blood versus endotracheal aspirate bacteriological findings. In their study this was a secondary endpoint, and the number of positive samples was too low to draw any conclusion. Yet the results are of note: several patients with negative blood cultures at baseline became positive for the same bacterial species found in the tracheal aspirate as soon as 5 minutes after intubation. Whether the species found in the blood were lung borne is not proven. However, the time course of blood culture is striking. The fact that a similar number of patients in the control and recruitment maneuver group became positive after intubation apparently favors the safety of the recruitment maneuver itself, as Constantin and colleagues discuss. Exacerbation of hypoxia at the time of intubation [7] or the interaction between hypoxia and mechanical ventilation [5], maybe even peri-intubation ventilation, cannot be excluded. Irrespective of the mechanism, this is an intriguing concept that deserves further insight, given its potential impact on outcome.

Whatever the contribution of recruitment maneuvers to the development of ventilator-induced lung injury, if any, data on their long-term effects on primary or secondary outcomes in patients with acute lung injury are lacking. What is given is that recruitment maneuvers do not simply improve oxygenation.
Abbreviations

$\mathrm{PaO}_{2}=$ arterial partial pressure of oxygen; $\mathrm{PEEP}=$ positive end-expiratory pressure.

\section{Competing interests}

The author declares that he has no competing interests.

Published: 1 July 2010

\section{References}

1. Constantin JM, Futier E, Cherprenet AL, Chanques G, Guerin R, CayotConstantin S, Jabaudon M, Perbet S, Chartier C, Jung B, Guelon D, Jaber S, Bazin JE: A recruitment maneuver increases oxygenation after intubation of hypoxemic intensive care unit patients: a randomized controlled study. Crit Care 2010, 14:R76.

2. Lachmann B: Open up the lung and keep the lung open. Intensive Care Med 1992, 18:319-321.

3. Foti G, Cereda M, Sparacino ME, De Marchi L, Villa F, Pesenti A: Effects of periodic lung recruitment maneuvers on gas exchange and respiratory mechanics in mechanically ventilated acute respiratory distress syndrome (ARDS) patients. Intensive Care Med 2000, 26:501-507.

4. Fan E, Wilcox ME, Brower RG, Stewart TE, Mehta S, Lapinsky SE, Meade MO, Ferguson ND: Recruitment maneuvers for acute lung injury: a systematic review. Am J Respir Crit Care Med 2008, 178:1156-1163.

5. Nahum A, Hoyt J, Schimitz L, Moody J, Shapiro R, Marini JJ: Effect of mechanical ventilation strategy on dissemination of intratracheally instilled Escherichia coli in dogs. Crit Care Med 1997, 25:1733-1743.

6. Lincy CY, Zhang H, Cheng KC, Slutsky AS: Mechanical ventilation may increase susceptibility to the development of bacteremia. Crit Care Med 2003, 31:1429-1434.

7. Gaffin SL, Brock-Utne JG, Zanotti A, Wells MT: Hypoxia-induced endotoxemia in primates: role of reticuloendothelial system function and anti-lipopolysaccharide plasma. Aviat Space Environ Med 1986, 57:1044-1049.

doi:10.1186/cc9042

Cite this article as: Valenza F: Do recruitment maneuvers simply improve oxygenation? Critical Care 2010, 14:173. 